\title{
Projeto de Antena Triangular Sierpinski para Bloqueadores de Telefones Celulares
}

\author{
Júlio C. B. D’Oliveira, Emmanuel A. Machado, Maurício H. C. Dias, José C. A. Santos ${ }^{1}$
}

\begin{abstract}
Resumo-Em aplicações de RF que demandam o uso simultâneo de duas ou mais bandas, antenas de geometria fractal podem ser uma solução conveniente. Neste contexto, o presente trabalho relata um projeto recente de antena fractal para uso em bloqueadores de telefones celulares. Partindo da síntese aproximada de uma monopolo Sierpinski, realizou-se uma análise complementar em software de modelagem eletromagnética (CST) variando-se diferentes parâmetros da antena para se chegar a uma configuração de melhor desempenho. Um protótipo foi montado e testado para validar a metodologia adotada.
\end{abstract}

Palavras-Chave-Antena triangular Sierpinski, antenas fractais, bloqueio de RF, telefonia celular.

Abstract-In multiband RF applications, fractal antennas may be a convenient solution. The present work summarizes a recent fractal antenna design, intended for $R F$ jamming of cellular phones. Starting from an approximate synthesis of a Sierpinski monopole, a complimentary analysis has been carried out in an electromagnetic modeling software tool (CST). Different antenna parameters have been varied in an attempt to reach a configuration with the best performance. A prototype was assembled and tested in order to validate the adopted procedure.

Index Terms-Sierpinski triangle antenna, fractal antennas, RF jamming, cellular phones.

\section{INTRODUÇÃO}

O bloqueio de sinais de RF, em especial os de ligações por telefones móveis, é uma aplicação que ganhou destaque nos últimos anos no cenário brasileiro. Talvez o maior responsável por isto tenha sido o uso freqüente de celulares em presídios por detentos, problema para o qual as autoridades de segurança pública ainda não conseguiram dar solução satisfatória definitiva. Mas este cenário não é o único em que bloqueadores de RF se fazem úteis ou necessários. Ainda na esfera de aplicações de segurança para a população em geral, destacam-se as operações de garantia da lei e da ordem (GLO), principalmente em cenários urbanos, como por exemplo: controle de distúrbios; ações anti-sequestro; intervenções contra o tráfico de drogas; etc. No contexto de

\footnotetext{
1 Secão de Engenharia Elétrica, Instituto Militar de Engenharia, Rio de Janeiro, Brasil. E-mails: juliocbueno@yahoo.com.br, emmanuel machado@yahoo.com.br, e \{mhcdias, araujo\}@ime.eb.br. Este trabalho foi parcialmente financiado pela FAPERJ (E-26/171.333/2006).
}

Defesa, por sua vez, o bloqueio de RF ou jamming é ação corrente prevista há muito tempo na doutrina de Guerra Eletrônica (GE), mais especificamente como opção de Medida de Ataque Eletrônico (MAE) [1]-[2].

Diante do natural apelo militar deste tema (com interesse explícito do Exército Brasileiro, através de seu Plano Básico de Ciência e Tecnologia - PBCT) e de seu forte atrativo dual, alguns trabalhos de pesquisa e desenvolvimento vêm sendo realizados recentemente dentro da linha de pesquisa de Eletromagnetismo Aplicado do IME. Dentre eles, destacamse: análise do bloqueio de terminais GPS [3]; projeto e testes de um bloqueador para telefones celulares IS-95 [4]; e considerações sobre o uso de arranjos de antenas em bloqueadores de banda dupla [5]. Este último denota uma preocupação atual com a necessidade de soluções multibanda, como no bloqueio de celulares, por exemplo, cujo espectro se divide (grosso modo) em duas faixas distintas centradas aproximadamente em 850 e $1850 \mathrm{MHz}$ (no Brasil).

Nesse contexto, uma opção relativamente recente que vem ganhando força é o uso de antenas fractais. Na verdade, a aplicação do conceito de geometrias fractais à síntese de antenas tem como principal atrativo a possibilidade de redução das dimensões da antena e da perda de retorno na faixa de interesse, além do aumento da largura de faixa. Entretanto, algumas configurações, como o triângulo Sierpinski, apresentam ainda o desejável comportamento multi-banda [6]-[8].

Tais atributos em potencial levaram os presentes autores a realizar um trabalho de investigação sobre a pertinência do uso de antenas Sierpinski em bloqueadores de telefones celulares, recém-concluído [9], cujos principais resultados são aqui descritos. Partindo de um triângulo Sierpinski com dimensões iniciais determinadas para a antena ressoar nas duas bandas celulares, realizou-se uma análise no software CST Microwave Studio (CST) variando-se diferentes parâmetros da antena, com o objetivo de se chegar a uma configuração de bom desempenho, com as ressonâncias coincidindo com as freqüências desejadas. Um protótipo artesanal foi montado e testado para validar a antena assim projetada.

Este artigo está organizado da seguinte forma. A seção II apresenta uma breve revisão bibliográfica sobre antenas fractais, com ênfase nos trabalhos que abordam o triângulo Sierpinski e suas variações. A metodologia adotada para o projeto da antena é descrita na seção seguinte. Na seção IV a análise paramétrica no CST e os testes com um protótipo montado são apresentados. Por fim, a seção V traz as considerações finais deste trabalho. 


\section{REVISÃo BIBLIOGRÁFICA}

\section{A. O Triângulo Sierpinski como Geometria Fractal}

O triângulo Sierpinski foi uma das primeiras geometrias fractais consideradas para aplicação em antenas, seja na forma de monopolo, seja na de dipolo [10]. Embora a geometria típica consista de triângulos eqüiláteros, a descrição se aplica a qualquer triângulo. A geração desta geometria pode se dar de duas formas distintas: por cópias múltiplas; ou por decomposição. A Fig. 1 ilustra esta última opção, na qual se começa com um triângulo maior, e em seguida une-se os pontos médios das três arestas para extrair um triângulo intermediário. $\mathrm{O}$ mesmo procedimento é repetido nos três sub-triângulos formados na etapa anterior, e assim sucessivamente. $O$ número de vezes que este processo é realizado é chamado de ordem (ou nível) de iteração fractal [7], [10]. Fractais "ideais", em teoria, são formados por um número infinito de iterações, mas na geração de estruturas práticas há sempre a necessidade de truncar o processo [10]. A geração por cópias múltiplas é basicamente o inverso da decomposição.

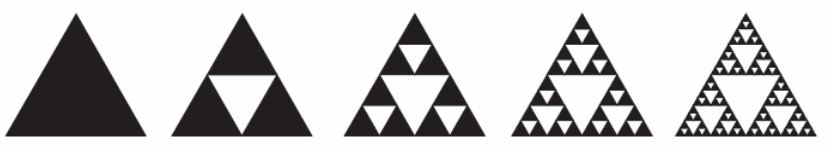

Fig. 1. Geração de triângulos Sierpinski por decomposição até 5 níveis.

A geração de geometrias fractais é fortemente baseada no uso de transformações afins (affine transformations), que envolvem escalonamento, rotação e translação [7]. Tais transformações podem ser expressas matematicamente como:

$$
W\left(\begin{array}{l}
x \\
y
\end{array}\right)=\left[\begin{array}{cc}
r \cos \theta & -s \cos \phi \\
r \operatorname{sen} \theta & s \operatorname{sen} \phi
\end{array}\right]\left(\begin{array}{l}
x \\
y
\end{array}\right)+\left(\begin{array}{l}
x_{0} \\
y_{0}
\end{array}\right)
$$

onde $r$ e $s$ são fatores de escala, $\theta$ e $\phi$ correspondem a ângulos de rotação, $x_{0}$ e $y_{0}$ são translações envolvidas na transformação. O triângulo da segunda iteração na Fig. 1 é obtido com três transformações, nas quais em todas $r=s=0,5, \theta=0$ e $\phi=90^{\circ}$, mas com diferentes pares de coordenadas de translação $\left(x_{0}, y_{0}\right)$ de modo a recompor o perímetro do triângulo original. $\mathrm{Na}$ iteração seguinte, as mesmas três transformações devem ser aplicadas a cada um dos três sub-triângulos (num total de nove), com as devidas alterações nas translações, e assim sucessivamente.

Se $r$ e $s$ forem ambas reduções ou ambas ampliações, a transformação é dita auto-afim. Se $r=s$ e $\theta=\phi \pm \pi / 2$, a transformação é auto-similar. A auto-similaridade, embora desejável, não é uma propriedade essencial para uma geometria ser considerada como fractal [7]. A Fig. 2 ilustra uma variação do triângulo Sierpinski não mais auto-similar, mas ainda assim fractal. Os efeitos da auto-similaridade (ou da sua ausência) no desempenho do triângulo Sierpinski como antena têm sido foco de investigação de diversos pesquisadores nos últimos anos, como resumido na breve revisão a seguir.

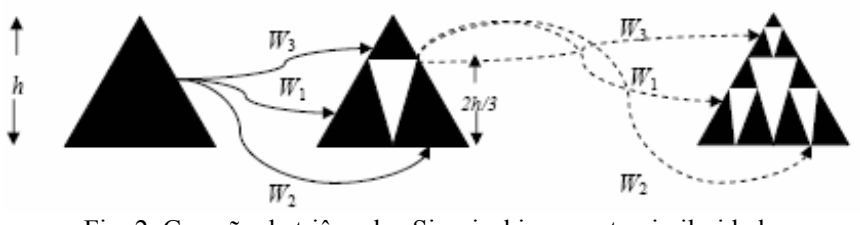

Fig. 2. Geração de triângulos Sierpinski sem auto-similaridade.

\section{B. Trabalhos Relacionados - Antenas Fractais}

Um dos trabalhos iniciais sobre antenas Sierpinski mais referenciados é o de Puente-Baliarda et al [10], em que os autores demonstram a característica multi-banda deste tipo de antena, a partir de simulações numéricas (baseadas no método das diferenças finitas no domínio do tempo - FDTD) e ensaios experimentais de um triângulo de ordem de iteração fractal 5. É importante ressaltar que o comportamento multibanda é caracterizado não só pela presença de múltiplas freqüências de ressonância ao longo do espectro, mas principalmente pelo comportamento similar naquelas freqüências no que se refere à distribuição espacial da radiação. Nesse sentido, os autores comparam o desempenho da fractal Sierpinski com antenas triangulares convencionais correlatas (bow-tie) para justificar seus argumentos. Seus resultados mostraram que as antenas bow-tie não podem ser consideradas como multi-banda no sentido estrito, pois elas apresentam diagramas de radiação distintos por faixa, apesar das múltiplas ressonâncias.

Ainda em [10], alguns resultados específicos interessantes podem ser destacados como apoio a projetos de antenas Sierpinski triangulares. A antena testada e montada era autosimilar, com fator de escala igual a $1 \frac{1}{2}$. Os autores observaram que a separação entre as freqüências de ressonância seguia aproximadamente este mesmo fator de escala, pelo menos a partir da terceira banda. Mais ainda, este comportamento não seria uma mera coincidência, mas sim uma conseqüência da auto-similaridade da geometria adotada. De modo geral, pode-se afirmar que a freqüência de operação de uma antena triangular Sierpinski é dada pela expressão:

$$
f_{n} \approx K \delta^{n} c / h
$$

onde $f_{n}$ é a n-ésima banda, $K$ é uma constante, $c$ é a velocidade da luz, $h$ é a altura da antena (como na Fig. 2), e $\delta$ é o inverso do fator de escala (no caso, $\delta=2$ ). A expressão é válida para $n>1$, e $K$ depende de propriedades dos materiais de confecção da antena (em [10], $K=0,13$ ). Aparentemente, quanto maior a permissividade e a espessura do substrato de sustentação da antena, menor $K$. Mesmo a primeira ressonância pode ser estimada a partir de (2), mas com outra constante $\left(K^{\prime}\right)$, que pode ser obtida da análise de antenas triangulares convencionais. Em [10], dois valores próximos foram apresentados $(0,17$ e 0,194$)$. Este comportamento distinto da primeira ressonância e outras distorções observadas no desempenho multi-banda (esperado em função da auto-similaridade) foram resultado essencialmente da truncagem das iterações fractais, segundo os autores.

Um trabalho posterior de Puente-Baliarda et al [11] apresentou uma análise (teórica e experimental) de versões distorcidas da Sierpinski eqüilátera de [10]. Os autores 
analisaram comparativamente o desempenho de mais dois triângulos, ambos isósceles, com ângulos de abertura de $30 \mathrm{e}$ $90^{\circ}$. As antenas foram montadas com os mesmos materiais e com a mesma altura da antena do trabalho anterior. Desse estudo, foi possível ainda acrescer o ângulo de abertura $(\alpha)$ como mais um parâmetro a (2), resultando em:

$$
f_{n} \approx 0,152 \cos (\alpha / 2) \delta^{n} c / h
$$

Uma conclusão importante em [11] se refere ao desempenho da antena mais estreita $\left(\alpha=30^{\circ}\right)$. Os autores observaram que a Sierpinski perde seu comportamento multibanda em termos de radiação, pois lobos laterais aparecem nas bandas mais altas. A explicação passa pelo fato que, quanto menor $\alpha$, mais parecida com uma monopolo linear a antena fica. A análise da distribuição de corrente na antena reforça este argumento. É como se ela operasse em dois modos: um modo Sierpinski, associado às ressonâncias da estrutura fractal; outro modo harmônico, associado às escalas maiores da antena. A superposição de ambos faz com que a corrente se distribua praticamente por toda a antena, mesmo em freqüências mais elevadas, diferentemente do que se observa na antena fractal com ângulo de abertura maior.

Vários trabalhos recentes sobre antenas fractais em geral são encontrados na literatura, como os de Gianvittorio [6] e Vinoy [7], por exemplo. Ambos analisaram diferentes tipos de geometria fractal, como monopolos de Koch, ilhas (laços) de Minkowsky, dipolos de árvore 2D e 3D, entre outros. Ambos também analisaram o triângulo Sierpinski em seus trabalhos e confirmaram o comportamento multi-banda já destacado por outros autores (como Puente-Baliarda [10]). Curiosamente, entretanto, o porquê deste comportamento peculiar de triângulos Sierpinski é motivo de discórdia até hoje. Puente-Baliarda [10] credita tal comportamento à estrutura fractal daquela geometria, e mais especificamente a sua auto-similaridade. Vinoy [7] contesta tal explicação, inicialmente com base em experimentos com versões do triângulo Sierpinski com número menor de iterações fractais (1 a 4), que mostraram o comportamento multi-banda mesmo no caso em que a antena era na verdade uma bow-tie. Sua argumentação foi reforçada posteriormente após testes com versões modificadas do triângulo Sierpinski em que a autosimilaridade era descaracterizada. Somente perturbações consideráveis na geometria afetaram o comportamento multibanda da Sierpinski. Outro trabalho nesta mesma linha chegou a conclusões similares, descaracterizando a autosimilaridade como explicação para o comportamento multibanda; o crédito iria para a periodicidade das lacunas ao longo do eixo central [12]. Gianvittorio [6], por sua vez, cita que nem mesmo carpetes (quadrados) Sierpinski, que são auto-similares, parecem gozar do mesmo atributo.

Dois resultados adicionais de Vinoy [7] em sua análise de triângulos Sierpinski merecem destaque por sua correlação com o presente artigo. O primeiro diz respeito aos efeitos do número de níveis de iteração fractal. De modo geral, as freqüências mais baixas são pouco perturbadas pela inserção de mais e mais lacunas triangulares fractais na estrutura (assumindo-se a estratégia de decomposição). As bandas mais altas parecem ser mais afetadas, especialmente quanto ao comportamento de radiação. A outra conclusão importante veio da análise da influência da escolha do substrato de suporte para a antena: quanto maior sua permissividade, menores as freqüências de ressonância.

Alguns trabalhos recentes têm buscado versões ainda mais compactas do triângulo Sierpinski que mantenham o comportamento multi-banda (pelo menos nas bandas de interesse). Em [13], por exemplo, alterações no terço superior do triângulo foram propostas e testadas tendo como foco a otimização do desempenho da antena especificamente nas duas bandas de telefonia celular. Já em [14], apenas metade de um triângulo foi utilizada, com ajustes adicionais na porção inferior da antena (ângulo de abertura) e no plano de terra. Mais uma vez, as alterações foram propostas buscando melhorar o desempenho nas faixas de operação desejadas.

Antenas fractais são um tema relativamente novo, e como tal, ainda pouco explorado no contexto nacional. Em um dos poucos trabalhos encontrados [15], os autores usam o método dos momentos (MoM) para calcular freqüências de ressonância e as respectivas impedâncias de entrada de um dipolo triangular Sierpinski com 4 iterações. A motivação do trabalho foi a possibilidade de uso de antenas fractais em rádios definidos por software (RDS). Em outro artigo de autores brasileiros [16], uma análise por simulação (MoM) completa (perda de retorno e diagrama de radiação) de monopolos espirais fractais é apresentada. Como resultados principais, verificou-se que as antenas fractais analisadas apresentaram as seguintes vantagens (em comparação a monopolos): redução de tamanho em baixas freqüências; e comportamento banda-larga em altas freqüências. Esses mesmos autores realizaram ainda outro trabalho com antenas fractais, mas com outra geometria [17]-[18], em que apresentam uma análise teórica, com apoio de simulação (MoM), do efeito da variação da dimensão fractal sobre diagramas de radiação de monopolos de Koch. Por fim, em [19] uma antena circularmente polarizada com borda fractal de Koch é proposta e analisada (MoM).

\section{PRoJeto DE UMA ANTENA TRIANGUlar SiERPINSKI}

Conforme destacado na introdução deste artigo, a premissa deste trabalho era projetar uma antena que apresentasse desempenhos similares nas duas faixas de freqüência da telefonia celular no Brasil. A Tabela I apresenta uma versão compacta da distribuição espectral (controlada e definida pela ANATEL) desse serviço no Brasil. Na aplicação desejada, o foco foi o bloqueio da recepção do terminal móvel. Com isso, definiu-se que a antena deveria apresentar freqüências de ressonância $f_{1}$ e $f_{2}$ coincidentes com as freqüências centrais do enlace direto, ou seja, 881,5 e 1827,5 MHz, respectivamente. As larguras de faixa mínimas necessárias seriam de 25 e 30 $\mathrm{MHz}$, respectivamente.

TABELA I

ESPECTRO DA TELEFONIA CELULAR NO BRASIL

\begin{tabular}{|c|c|c|}
\hline Denominação das & \multicolumn{2}{|c|}{ Faixas de operação [MHz] } \\
\cline { 2 - 3 } Bandas & Uplink (enlace reverso) & Downlink (enlace direto) \\
\hline A, B & $824-849$ & $869-894$ \\
C, D, E & $1710-1755$ & $1805-1850$ \\
\hline
\end{tabular}


Uma característica interessante do requisito frequencial em questão é que as bandas apresentam uma relação $\Delta f=f_{2} / f_{1}=2,07$, ou seja, praticamente a mesma relação de escala observada nas bandas superiores da Sierpinski triangular $(\delta=2)$. Em função disso, a idéia neste trabalho foi projetar uma Sierpinski em que a segunda e terceira ressonâncias fossem justamente as bandas $f_{1}$ e $f_{2}$ desejadas. Para tal, no dimensionamento inicial da antena, assumiu-se um valor aproximado para a primeira ressonância $\left(f_{0}\right)$ em função dos resultados apresentados em [10]. Mais especificamente, em função da relação $f_{1} / f_{0}$ (igual a 3,5 para a antena analisada naquela referência), e dos valores de $K$ para primeira ressonância $(1,7$ e 1,94). A primeira banda ficou, portanto, na parte mais alta de VHF, em torno de $275 \mathrm{MHz}$. A Fig. 3 ilustra a geometria adotada no projeto, que corresponde a um triângulo Sierpinski com 3 iterações fractais. Em função daquelas considerações sobre a determinação de $f_{0}$, assumiuse como valor de partida para a altura $h=19 \mathrm{~cm}$. As alturas intermediárias $h$ ' e $h$ " foram calculadas usando-se a relação de escala $\Delta f=2,07 \approx \delta=2\left(h^{\prime}=9,17\right.$ e $\left.h^{\prime \prime}=4,42 \mathrm{~cm}\right)$.

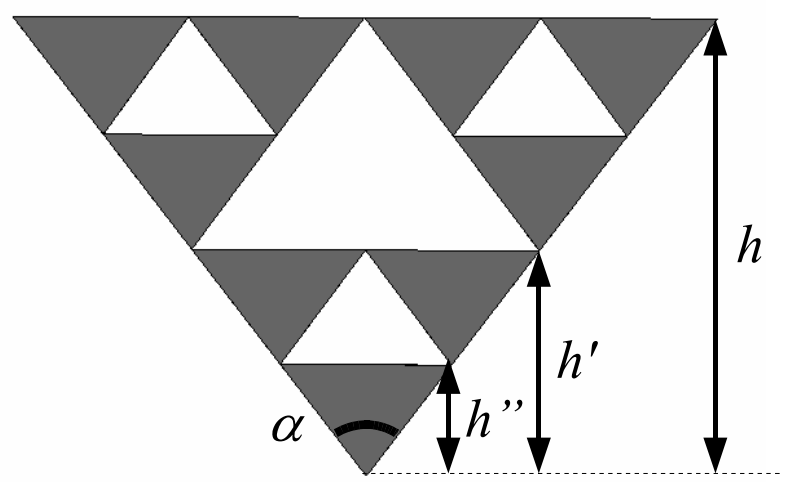

Fig. 3. Geometria da antena projetada.

A antena assim dimensionada foi analisada na ferramenta de simulação de campos eletromagnéticos CST Microwave Studio. A Fig. 4 reproduz o desenho realizado naquela plataforma. Observa-se que uma alimentação coaxial foi assumida, como indicado no detalhe da Fig. 4. O desempenho obtido por esta versão inicial da antena não foi satisfatório, em particular pelo desvio grande na segunda faixa de ressonância, que caiu em $1908 \mathrm{MHz}(80,5 \mathrm{MHz}$ acima da desejada). Em função disso, resolveu-se testar diferentes configurações da antena, alterando os valores de alguns parâmetros, na busca por uma versão com desempenho mais próximo do desejado.
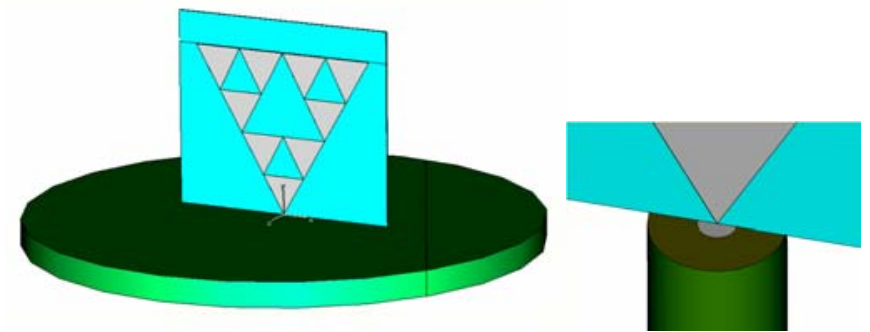

Fig. 4. Antena simulada no CST - o detalhe indica a alimentação coaxial.

\section{ANÁlise PARAMÉtrica E TeStes EXPERIMENTAIS}

$\mathrm{Na}$ busca por um melhor dimensionamento da antena, procurou-se analisar a sensibilidade de desempenho a três parâmetros específicos, a saber: permissividade relativa do dielétrico de suporte da antena $\left(\varepsilon_{r}\right)$; espessura deste mesmo substrato $\left(h_{s}\right)$; e ângulo de abertura do triângulo Sierpinski $(\alpha)$. Na presente análise, utilizando-se os recursos de parametrização e otimização do CST, como o foco era justamente coincidir a segunda e terceira ressonâncias da antena com as duas faixas desejadas, adotou-se como função objetivo justamente este critério.

A primeira rodada de testes verificou a influência da escolha do substrato de suporte da antena. Para tal, alguns parâmetros foram fixados, a saber: $h_{s}=1,65 \mathrm{~mm} ; \alpha=60^{\circ}$. Em função do uso da otimização previamente citada, algumas dimensões eram modificadas pelo algoritmo do CST a cada análise. Mais especificamente, a altura da antena $(h)$ era alterada automaticamente a cada teste. As demais alturas foram parametrizadas a $h$ pela relação $\Delta f$. Cinco diferentes valores de permissividade foram testados - os resultados estão resumidos na Tabela II. Observa-se que o desempenho em termos de casamento de impedância melhora com substratos de maior permissividade. A mesma tendência foi observada quanto à influência da espessura do dielétrico: quanto maior $h_{s}$, menores as perdas de retorno $\left(\mathrm{S}_{11}\right)$ nas ressonâncias.

TABELA II

RESULTADO DAS SIMULAÇÕES VARIANDO-SE A PERMISSIVIDADE DO DIELÉTRICO DO SUBSTRATO SUPORTE $\left(\varepsilon_{R}\right)$

\begin{tabular}{|c|c|c|c|c|c|}
\hline$\varepsilon_{r}$ & $h[\mathrm{~mm}]$ & $f_{1}[\mathrm{MHz}]$ & $\mathrm{S}_{11}[\mathrm{~dB}]$ & $f_{2}[\mathrm{MHz}]$ & $\mathrm{S}_{11}[\mathrm{~dB}]$ \\
\hline 1 & 200 & 866 & $-15,05$ & 1832 & $-7,10$ \\
2 & 196 & 868 & $-16,33$ & 1818 & $-7,63$ \\
3 & 194 & 868 & $-16,70$ & 1824 & $-9,37$ \\
4 & 189 & 878 & $-23,84$ & 1826 & $-9,36$ \\
5 & 187 & 880 & $-26,60$ & 1816 & $-9,59$ \\
\hline
\end{tabular}

Os resultados da análise de sensibilidade à variação de $\alpha$ não foram tão homogêneos quanto os da rodada anterior, como pode ser constatado na Tabela III. Aqui, o parâmetro fixado foi $\varepsilon_{r}=4,36$ (valor da permissividade relativa de uma placa de fibra de vidro); a espessura foi mantida em 1,65 mm (também associada à placa disponível). O desempenho quando $\alpha=30^{\circ}$ foi bem pior que o do projeto inicial, como esperado, pois vai ao encontro do que já fora constatado em [11]. À medida que a abertura aumenta, o casamento também aumenta, pelo menos até algum valor em torno de $75^{\circ}$.

TABELA III

RESULTADO DAS SIMULAÇÕES VARIANDO-SE O ÂNGULO DE ABERTURA DO TRIÂNGULO $(\alpha)$

\begin{tabular}{|c|c|c|c|c|c|}
\hline$\alpha$ [graus] & $h[\mathrm{~mm}]$ & $f_{1}[\mathrm{MHz}]$ & $\mathrm{S}_{11}[\mathrm{~dB}]$ & $f_{2}[\mathrm{MHz}]$ & $\mathrm{S}_{11}[\mathrm{~dB}]$ \\
\hline 30 & 205,0 & 880 & $-10,30$ & 1970 & $-3,37$ \\
45 & 198,5 & 878 & $-12,81$ & 1830 & $-6,15$ \\
60 & 187,7 & 881 & $-26,00$ & 1820 & $-8,85$ \\
75 & 173,0 & 882 & $-15,00$ & 1826 & $-28,50$ \\
90 & 155,5 & 882 & $-8,20$ & 1824 & $-11,20$ \\
\hline
\end{tabular}

Com base nos resultados da análise paramétrica, e tendo como restrições os materiais disponíveis em laboratório para montagem de um protótipo artesanal, foi feito um último teste no CST da configuração que seria montada. Os parâmetros 
desta versão final foram os seguintes: $\alpha=75^{\circ} ; \varepsilon_{r}=4,36$; $h_{s}=1,65 \mathrm{~mm} ; h=17,3 \mathrm{~cm}$. A Fig. 5. apresenta a resposta da perda de retorno, e a Fig. 6 os diagramas de radiação nas duas bandas de ressonância. Em $f_{1}$ a largura de faixa ficou em 52 $\mathrm{MHz}$, enquanto que em $f_{2}$ foi de $124 \mathrm{MHz}$, ou seja, bem maiores que o desejado para as bandas A-B e C-E, respectivamente. O comportamento em termos de radiação também foi aceitável, pois foi similar nas duas bandas, como pode ser constatado na Fig. 6.

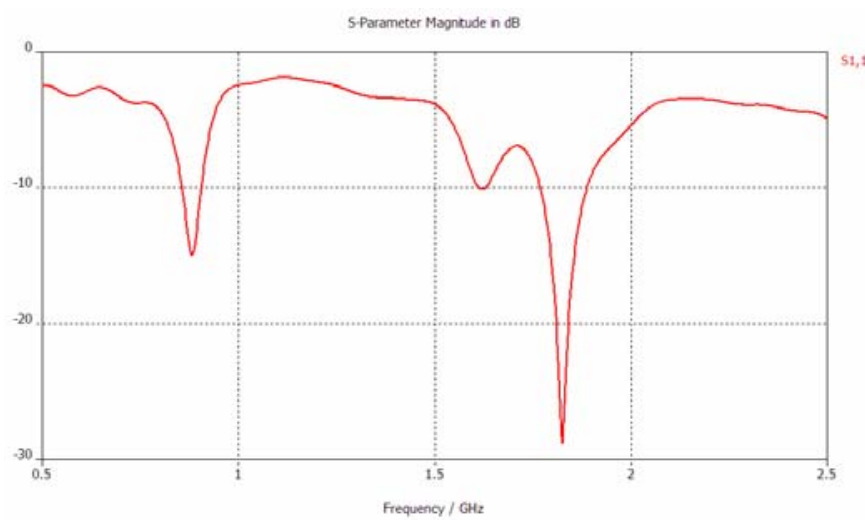

Fig. 5. Perda de retorno da configuração final da antena.
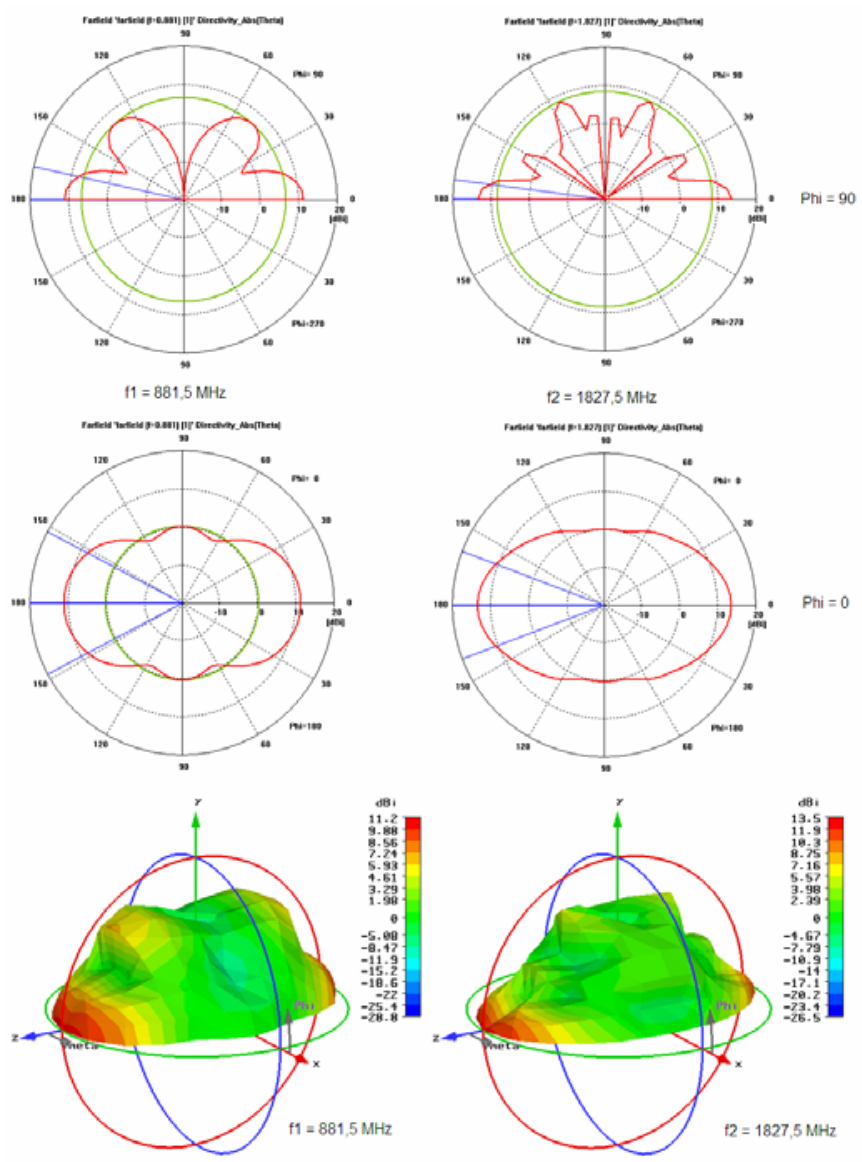

Fig. 6. Diagramas de radiação da configuração final da antena.

Para a montagem da antena Sierpinski, foi utilizada uma placa de fibra de vidro dupla face de $21 \times 29,7 \mathrm{~cm}^{2}$. Para o plano de terra, uma placa de fenolite face única de
$40 \times 40 \mathrm{~cm}^{2}$. Um conector SMA de $50 \Omega$ foi utilizado para alimentação da antena (condutor interno soldado à ponta inferior do triângulo; condutor externo soldado ao plano de terra). A Fig. 7 traz uma foto do protótipo artesanal final.

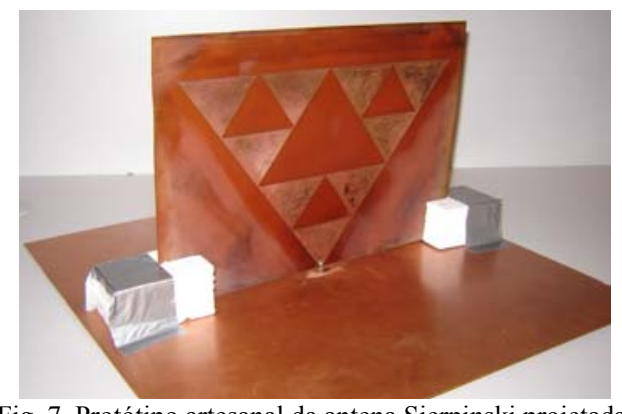

Fig. 7. Protótipo artesanal da antena Sierpinski projetada.

Testes foram realizados no laboratório de RF do IME para comparação com o desempenho previsto pela análise no CST. A perda de retorno foi medida em um Analisador de Redes E5070B da Agilent (300 kHz a $3 \mathrm{GHz}$ ), e a Fig. 8 apresenta a resposta obtida. A segunda ressonância coincidiu com a projetada, mas verificou-se um desvio considerável na freqüência da banda inicial, para $844 \mathrm{MHz}$, e as perdas de retorno foram piores que as esperadas em ambas as bandas, especialmente na primeira $\left(\mathrm{S}_{11}\right.$ não chegou a $\left.-10 \mathrm{~dB}\right) . \mathrm{O}$ diagrama de radiação foi testado apenas qualitativamente, procurando verificar se os máximos de radiação ocorriam nas direções de broadside da antena ( 0 e $\left.180^{\circ}\right)$, o que se confirmou.

O resultado pouco satisfatório dos testes pode ser creditado a alguns fatores. Primeiramente, devido ao próprio processo artesanal de montagem, com várias imprecisões. Por exemplo, a altura foi $5 \mathrm{~mm}$ menor que a dimensionada. $\mathrm{O}$ processo de corrosão apresentou várias falhas, removendo parte do metal da antena. As conexões entre os sub-triângulos ficaram com espessuras assimétricas, entre outros detalhes. Em segundo lugar, pelo fato do laboratório não dispor de condições ideais para testes com antenas, que sofrem a influência de reflexões e espalhamentos eletromagnéticos. De qualquer forma, dadas todas as limitações citadas, ao menos qualitativamente o teste foi válido.

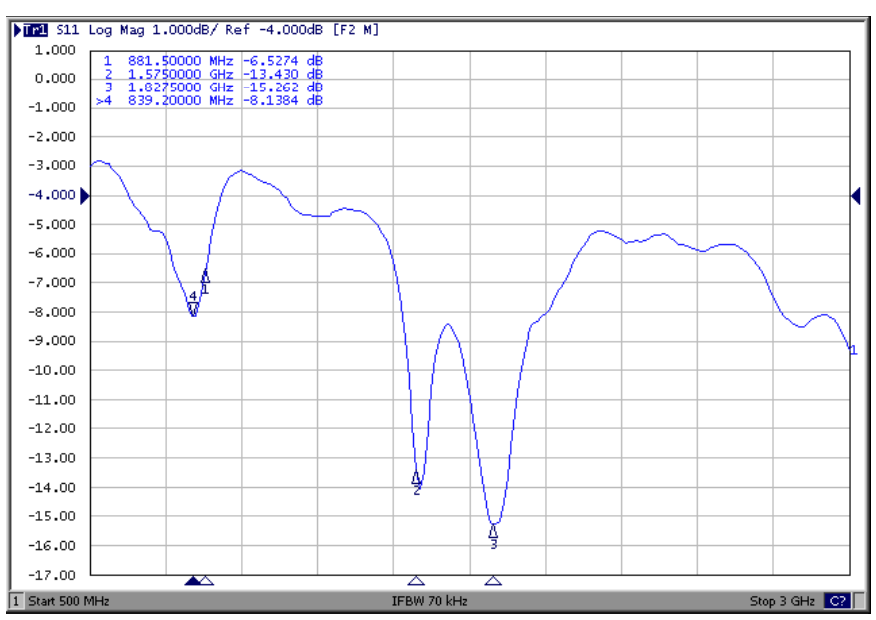

Fig. 8. Perda de retorno medida da antena Sierpinski montada. 


\section{COMENTÁRIOS FINAIS}

O presente trabalho descreveu um projeto de antena para uso em bloqueadores de telefones celulares. A geometria escolhida foi o triângulo Sierpinski, uma estrutura fractal bastante citada na literatura atual, justamente por ter um comportamento multi-banda. Nesse contexto, uma breve revisão bibliográfica sobre antenas fractais foi apresentada, com foco na geometria triangular Sierpinski. Alguns resultados relevantes para projetos deste tipo de antena foram destacados, e serviram de base para o trabalho aqui descrito.

A proximidade entre a razão das duas bandas desejadas e o fator de escala próprio de um triângulo Sierpinski torna esta geometria uma candidata natural para a aplicação em questão. Feita esta opção, a metodologia de projeto adotada foi o dimensionamento inicial da antena a partir das freqüências de operação desejadas, seguido de uma análise paramétrica em ferramenta de simulação de campos eletromagnéticos, para buscar uma configuração de melhor desempenho.

A partir dos resultados da análise, chegou-se a uma configuração final para a antena que foi mais uma vez analisada por simulação numérica. Esta última versão foi então montada e testada com relativo sucesso. O processo de montagem artesanal e as condições impróprias do local de teste foram fatores que contribuíram negativamente.

\section{REFERÊNCIAS}

[1] M. R. Frate, M. Ryan, Electronic Warfare for the Digitized Battlefield, Artech House, 2001.

[2] R. A. Poisel, Modern Communication Jamming Principles and Techniques, Artech House, 2004.

[3] C. R. M. Souza, M. H. C. Dias, J. C. A. Santos, "Análise da Vulnerabilidade de Receptores GPS Comerciais sob Ação de Interferência Intencional", in VIII Simpósio de Guerra Eletrônica (VIII SIGE), São José dos Campos - SP, 21-23 Nov. 2006, pp. 1-4.

[4] R. S. Toscano, M. H. C. Dias, J. C. A. Santos, "Análise da eficácia de um bloqueador de RF: estudo de caso para terminais IS-95", in $X X V$ Simpósio Brasileiro de Telecomunicações (SBrT'07), Recife - PE, 3-6 Set. 2007, pp. 1-5.

[5] D. S. V. Araujo, J. C. A. Santos, M. H. C. Dias, “A dual band steerable cell phones jammer", in SBMO/IEEE MTT-S International Microwave and Optoelectronics Conference (IMOC 2007), Salvador - BA, 29 Out - 01 Nov. 2007, pp. 611-615.

[6] J. Gianvittorio, Y. Rahmat-Samii, "Fractal Antennas: A Novel Antenna Miniaturization Technique, and Applications", IEEE Antennas and Propagation Magazine, vol. 44, no. 1, pp. 20-36, Fev. 2002.

[7] K.J. Vinoy, Fractal Shaped Antenna Elements for Wide- and MultiBand Wireless Applications, tese de doutorado, Pennsylvania State University, EUA, Ago. 2002.

[8] C. A. Balanis, Antenna Theory: Analysis and Design, $3^{\text {rd }}$ ed, Wiley, 2005.

[9] J. C. B. D’Oliveira, E. A. Machado, Antena Fractal Tipo Sierpinski Aplicada em Bloqueadores de Aparelhos de Telefonia Celular, monografia de projeto de fim de curso, Instituto Militar de Engenharia, Rio de Janeiro, Ago. 2007.

[10] C. Puente-Baliarda, J. Romeu, R. Pous, A. Cardama, "On the Behavior of the Sierpinski Multiband Fractal Antenna", IEEE Transactions on Antennas and Propagation, vol. 46, no. 4, pp. 517-524, Abr. 1998.

[11] C. Puente-Baliarda, C. B. Borau, M. N. Rodero, J. R. Robert, "An Iterative Model for Fractal Antenas: Application to the Sierpinski Gasket Antenna", IEEE Transactions on Antennas and Propagation, vol. 48, no. 5, pp. 713-719, Maio de 2000.

[12] S. R. Best, "On the Significance of Self-Similar Fractal Geometry in Determining the Multiband Behavior of the Sierpinski Gasket Antenna", IEEE Antennas and Wireless Propagation Letters, vol. 1, pp. 22-25, 2002.
[13] G. F. Tsachtsiris, C. F. Soras, M. P. Karaboikis, V. T. Makios, "Analysis of a Modified Sierpinski Gasket Monopole Antenna Printed on Dual Band Wireless Devices", IEEE Transactions on Antennas and Propagation, vol. 52, no. 10, pp. 2571-2579, Out. 2004.

[14] K. C. Hwang, "A Modified Sierpinski Fractal Antenna for Multiband Application”, IEEE Antennas and Wireless Propagation Letters, vol. 6, pp. 357-360, 2007.

[15] A. G. M. Lima, L. R. A. X. Menezes, "A Multiband Fractal Antenna Array to Software Defined Radio", in SBMO/IEEE MTT-S International Microwave and Optoelectronics Conference (IMOC 2005), Brasília - DF, 25-28 Jul. 2005, pp. 256-258.

[16] K. Q. Costa, V. Dmitriev, C. Rodrigues, "Fractal Spiral Monopoles: Theoretical Analysis and Bandwidth Optimization", in SBMO/IEEE MTT-S International Microwave and Optoelectronics Conference (IMOC 2005), Brasília - DF, 25-28 Jul. 2005, pp 650-653.

[17] K. Q. Costa, V. Dmitriev, "Theoretical Analysis of a Modified Koch Monopole with Reduced Dimensions", IEE Proceedings on Microwaves, Antennas and Propagation, vol. 153, pp. 475-479, 2006.

[18] K. Q. Costa, V. Dmitriev, "Estudo Teórico de Monopolos de Koch Modificados com Dimensões Reduzidas", in $11^{\circ}$ Simpósio Brasileiro de Microondas e Optoeletrônica / $6^{\circ}$ Congresso Brasileiro de Eletromagnetismo (MOMAG 2004), São Paulo - SP, 16-19 Ago. 2004, pp. 1-5.

[19] P. N. Rao, N. V. S .N Sarma, "Koch Fractal Boundary Single Feed Circularly Polarized Microstrip Antenna", Journal of Microwaves, Optoelectronics and Electromagnetic Applications, vol. 6, no. 2, pp. 406-413, Dez. 2007. 